\title{
ESCOLHA DO MATERIAL DA TELA PARA DISPOSIÇÃO INTRA- PERITONEAL NA CORREÇÃO CIRÚRGICA DE DEFEITOS HERNIÁRIOS DA PAREDE ABDOMINAL
}

\author{
The choice of the mesh composition to use in the intraperitoneal position in the surgical repair \\ of abdominal wall defects
}

Ubirajara Rutilio Mendes e Ferreira de ARAÚJO, Nicolau Gregori CZECZKO, Andressa DEALLARMI, Flávia Emilie HEMOVISKI, Helena V. C. Pereira ARAÚJo

Trabalho realizado na Faculdade Evangélica do Paraná - FEPAR e Instituto de Pesquisas Médicas - IPEM, Curitiba, PR, Brasil.

DESCRITORES - Telas cirurgicas. Hérnia. Parede abdominal. Aderências teciduais.
RESUMO - Introdução - O uso de telas para a correção cirúrgica de defeito herniários da parede abdominal contribuiu para a acentuada redução dos índices de recidiva das hérnias. Contudo, o seu uso intra-abdominal cursa com a formação de aderências e diversas complicações, dentre elas a obstrução intestinal. Método - Foram selecionados, nos bancos de dados PubMed e Medline, 27 artigos científicos atualizados e com informações relevantes à respeito das propriedades mais importantes das telas (material, diâmetro dos poros, estrutura tridimensional e peso molecular) para colocação intra-abdominal, evitando-se a formação de aderências e recidiva da hérnia. Conclusão - Atualmente as telas de dupla-composição são as mais indicadas para uso intra-peritoneal. Estas têm uma face composta por um material (preferencialmente macro poroso e reticular) que fica em contato com a musculatura e tem o objetivo de manter a resistência tênsil da parede abdominal. A outra face, composta por outro tipo de material (preferencialmente micro poroso e laminar), deve ficar em contato com os órgãos intra-abdominais, porém sem induzir a formação de aderências

\section{Correspondência:}

Ubirajara R. M. e F. de Araújo

e-mail: biraraujo@bol.com.br

Fonte de financiamento: não há

Conflito de interesses: não há

Recebido para publicação: 21/10/2009

Aceito para publicação: 03/02/2010

HEADINGS - Surgical mesh. Hernia. Abdominal wall. Tissue adhesions.
ABSTRACT - Introduction - The use of meshes to surgical repair of abdominal wall defects has contributed increasingly to reduce the number of incisional hernias. This use, however, in the intraperitoneal position leads to adhesion formation and a great number of complications, as bowel obstruction. Methods - Twenty seven articles were selected from PubMed and Medline publications with important information about the meshes properties (material, pore size, spacial surface area, molecular weight) and its alocation in intraperitoneal position, preventing adhesion formation and incisional hernia. Conclusion - Nowadays, the composite meshes are the most specified to use in the intraperitoneal position. They are composed in one side with a material (macroporous and reticular) that must be in contact with the muscle and has the purpose to maintain the abdominal wall resistence. In the other side, another kind of material (microporous and laminar) that must be in contact with the intra-abdominal organs, however without inducing adhesion formation.

\section{HISTÓRICO}

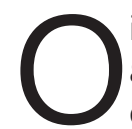

interesse e estudo das hérnias da parede abdominal é muito antigo; as primeiras referências sobre o assunto são da civilização egípcia e estão contidas no Papiro de Ebers (1536 a.C. $)^{19}$. Atribuise a Galeno (129-201 d.C.) a observação e correlação entre o surgimento de uma hérnia e a fraqueza da musculatura da parede abdominal ${ }^{22}$. A mais recente revolução para o tratamento cirúrgico das hérnias surgiu com Liechtenstein no início da década de 70; desenvolveu e difundiu o conceito da cirurgia sem tensão através do uso de tela de polipropileno. A partir de então houve início do emprego de próteses com o objetivo de reforçar a parede abdominal e o uso das telas difundiu-se de forma rápida 22. 
INTRODUÇÃO

O advento de materiais protéticos, telas das mais diversas composições, foi fundamental para melhora nos resultados técnicos da correção cirúrgica de defeitos congênitos ou adquiridos da parede abdominal. O seu uso, em comparação à simples rafia do defeito aponeurótico, reduziu consideravelmente os índices de recidivas das hérnias ${ }^{3,7,11,13}$. Estes valores variam de casuística para casuística, mas supõe-se que o uso de telas reduz a formação de hérnia incisional de cerca de $30-40 \%{ }^{2,18}$ para $5-10 \%{ }^{11}$. Conforme vários autores, dentre as próteses sintéticas atualmente disponíveis em nosso meio, a tela de polipropileno ainda é o material mais utilizado $2,13,16,17,24$.

O uso intra-abdominal de telas, contudo, tem indicações restritas, pois a colocação intra-peritoneal destas próteses apresenta complicações, podendo cursar com a formação de aderências, fístulas êntero-cutâneas ${ }^{9,11,12,13,14,18,23}$ e até mesmo oclusão intestinal $11,13,18,23$, sendo esta última, a complicação mais séria da formação de aderências ${ }^{10,21}$. A obstrução intestinal apresenta quadro clínico que acarreta alta taxa de morbi-mortalidade para os pacientes, bem como, custos altos para o sistema de saúde pública. Em decorrência disto, atualmente há procura incessante por uma composição de tela que traga as vantagens das já existentes, mantendo a resistência e força tênsil dos tecidos, porém sem provocar as suas diversas complicações ${ }^{6}$.

A recente utilização de telas de dupla composição com as chamadas barreiras anti-aderência vem ao encontro de soluções a essas complicações, isto é, procura proporcionar adequada força tênsil aos tecidos sem, contudo, provocar a formação de aderências e seus efeitos deletérios?

\section{Mecanismo de formação das aderências}

A formação de aderências faz parte do processo natural da cicatrização e ocorre em $90-100 \%$ dos procedimentos cirúrgicos ${ }^{8,25}$. O trauma cirúrgico causa formação de exsudato de fibrina, esta por sua vez, forma aderências temporárias até que o sistema fibrinolítico as absorva com a ajuda do ativador de plasminogênio. Porém, esta absorção é retardada ou inibida na presença de isquemia, inflamação ou corpo estranho; isto causa redução na fibrinólise, o que leva a aumento na deposição de fibrina. A matriz de fibrina gradualmente é invadida por fibroblastos, macrófagos e vasos sanguíneos, permitindo que esta se organize, e em cerca de cinco dias se transforme em aderências tissulares ${ }^{8,25}$.

\section{MÉTODO}

Foi realizado levantamento bibliográfico nos bancos de dados PubMed e Medline, selecionando-se
27 artigos científicos atualizados e com informações relevantes à respeito do uso de telas intra-abdominais para a correção de defeito da parede abdominal e suas propriedades principais (material, diâmetro dos poros, estrutura tridimensional e peso molecular) para diminuir o índice de formação de aderências, sem contudo propiciar a recidiva da hérnia.

\section{Propriedades da prótese e sua influência na cicatrização \\ Os diversos estudos neste campo de trabalho} demonstraram que não só o material que compõe a tela é importante no quesito formação de aderências, mas também o diâmetro de seus poros, a sua estrutura espacial tridimensional e o seu peso molecular. Todos estes dados devem ser pesquisados quando a tela for disposta no interior da cavidade peritoneal e, portanto, em contato diretos com vísceras, para evitar a sua adesão a estas estruturas e complicações indesejadas no futuro. Através destes novos conceitos, iniciou-se a confecção de telas chamadas de dupla composição (Figura 1), que têm como objetivo possuir duas faces de diferentes composições. Uma delas fica em contato com a musculatura, induzindo reação inflamatória capaz de manter a força tênsil dos tecidos e evitar a recidiva da hérnia. A outra, fica em contato direto com as vísceras e deve evitar a formação de aderências ${ }^{7}$. Como exemplos destes tipos de telas tem-se Parietex ${ }^{\circledR}$ e PTFE (teflon).

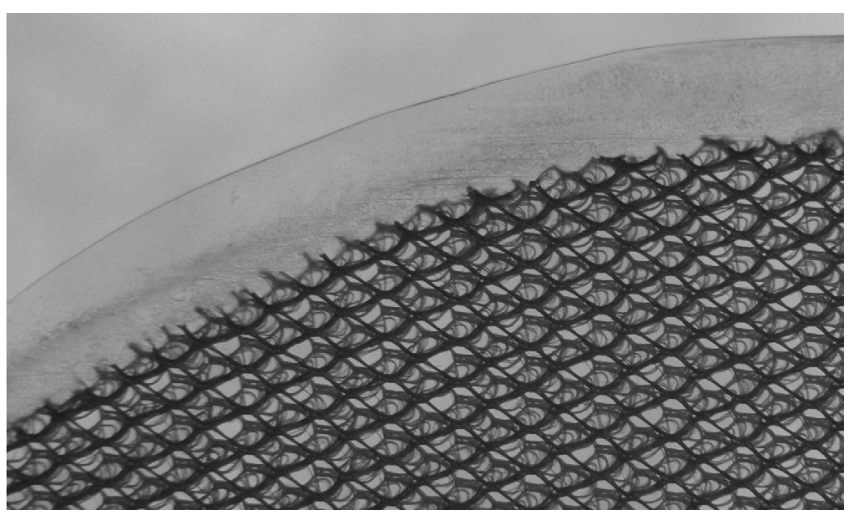

FIGURA 1 - Modelo esquemático de tela de dupla composição - a face superior (micro porosa / laminar) deve ficar em contato com as vísceras e a face inferior (macro porosa / reticular) deve ficar em contato com a musculatura.

\section{Material da prótese}

Sabe-se que o material que compõe a tela é importante devido a ser este um dos fatores que propicia a reação inflamatória e posteriormente e fibrose local. Os materiais absorvíveis de origem animal como xenoenxertos ou enxertos autólogos são melhores do que os materiais sintéticos porque induzem a cicatrização pela transformação da matriz celular ao invés de causar reação de corpo estranho (como as prótese sintéticas), o 
que diminui o risco de infecção ${ }^{14}$. Com isto, este tipo de material, quando provindo de animais, não tem o objetivo de propiciar resistência tênsil, pois sofre dissolução do tecido devido à resposta do hospedeiro ${ }^{23}$, mas serve para diminuir a formação de aderência e é por isto que muitas telas de dupla composição possuem colágeno bovino ou suíno na face que fica em contato com as vísceras. A composição do material também é importante porque a tela deve ser resistente à fadiga e capaz de suportar a pressão da parede abdominal, próteses de aço e titânio se fragmentam o que pode levar à recidiva da hérnia ${ }^{12}$ e erosão para a pele causando dor local ${ }^{14}$, por isto foram gradativamente abandonadas. As telas sintéticas absorvíveis (ácido poliglicólico, poliglactina) têm utilidade para reparo das hérnias por período curto porque induzem pouca reação inflamatória antes de sua hidrólise ${ }^{16}$ e têm sua indicação principal quando há presença de infecção. Finalmente, as telas sintéticas inabsorvíveis, feitas principalmente à base de polímeros (polipropileno, poliéster), propiciam adequado aporte de células inflamatórias e fibrose, sendo úteis na manutenção da resistência tênsil do tecido, porém com risco elevado de formação de aderências quando em contato com as vísceras.

\section{Diâmetro dos poros da prótese}

No que diz respeito à porosidade do material, ele é importante na integração dos tecidos. As telas micro porosas (celulose, colágeno, poliuretano), induzem levemente à colonização celular, reação inflamatória, e consequentemente à formação de aderências ${ }^{26,29}$. Os micro poros destas telas - menores de $10 \mu \mathrm{m}^{8}$-, induzem inadequada reação inflamatória, com pequeno aporte de tecido fibrocolagenoso e menor depósito de colágeno $0^{11,24}$. Por isto, estes materiais tendem a ser encapsulados e não infiltrados pelos tecidos ${ }^{18}$ e devem compor a face que fica em contato com as vísceras. Em contrapartida, as próteses macro porosas (polipropileno, poliéster) devem ficar em contato com a musculatura para atuar com o objetivo de manter a resistência e força tênsil da parede, isto ocorre porque a colonização celular e a reação inflamatória estão diretamente relacionadas à porosidade do material $5,6,7,29$. As telas macro porosas possuem poros maiores do que $75 \mu \mathrm{m}$, que é o tamanho que permite a migração de macrófagos, fibroblastos, fibras de colágeno e vasos sanguíneos ${ }^{4,26}$. Isto permite que a prótese seja infiltrada por estas células e fique intimamente aderida aos tecidos que a cercam.

\section{Estrutura tridimensional da prótese}

Com relação à estrutura espacial da tela, observouseem diversos estudos através da microscopia eletrônica, que as telas micro porosas têm estrutura mais laminar, o que permite depósito mais regular e organizado das células mesoteliais, permitindo a formação de um neo-peritônio nos primeiros dias após a sua colocação e, assim, diminuindo a formação de aderências ${ }^{5,6,9,13}$. $\mathrm{Na}$ prótese laminar ocorre mesotelização de forma simétrica e regular ao passo que na reticular o depósito do mesotélio é irregular e desorganizado, propiciando formação de aderências nos locais descobertos por mesotélio ${ }^{6,9,13}$. Fica claro, então, que a face laminar da tela deve ficar disposta para o interior da cavidade, isto é, em contato com as vísceras.

\section{Peso molecular da prótese}

O peso molecular, ou gramatura, de uma tela é definido pelo cálculo do seu peso, em gramas, dividido pela sua superfície, em metros quadrados, $\left(\mathrm{g} / \mathrm{m}^{2}\right)^{27}$. Isto é importante porque, segundo alguns autores ${ }^{1,15,20,27}$, as telas de baixo peso molecular (menos de $40 \mathrm{~g} / \mathrm{m}^{2}$ ) apresentam melhor biocompatibilidade e isto reduz as queixas de dor e desconforto no pós-operatório. Este mecanismo é desencadeado pela menor reação de corpo estranho das telas de baixo peso molecular ${ }^{15}$. Para que isto ocorra, estas próteses geralmente tendem a ser macro porosas e compostas com polímeros como o polipropileno entremeado por material absorvível como a poliglactina ${ }^{15,20,27}$ ou poliglecaprone ${ }^{15}$. Os poros alargados e a reabsorção parcial da tela permitem o acúmulo de gordura, ao invés de tecido fibrótico, entre as áreas de formação de granulomas, propiciando maior elasticidade aos tecidos ${ }^{28}$. Porém, alguns estudos demonstram que devido a estes fatores, há também propensão a um aumento no índice de recidiva das hérnias corrigidas com este tipo de tela ${ }^{20}$. Por isto, as telas de baixo peso molecular requerem maior número de estudos. Até o momento verificou-se que a composição e diâmetro dos poros da tela são mais importantes do que a sua gramatura ${ }^{15}$.

\section{CONCLUSÃO}

A escolha do material protético a ser usado deve ser feita de forma bastante criteriosa para evitar a recidiva da hérnia ou a formação de aderências e suas complicações. Com os conhecimentos atuais, pode-se dizer que embora as próteses de dupla-composição apresentem custos elevados, estes se justificam em relação aos benefícios que podem propiciar ao evitar as complicações que podem advir pela formação de aderências entre a tela e as vísceras. As chamadas barreiras anti-aderência, quando compostas por material absorvível, devem perdurar por no mínimo uma semana, que é o período de formação das aderências ${ }^{7}$.

Ainda não há consenso sobre qual é o melhor material a ser utilizado para a composição das próteses que devem ficar dispostas no interior da cavidade abdominal e em contato direto com vísceras, estudos futuros associados ao acompanhamento clínico a longo prazo de casos selecionados permitirão responder esta questão. Mas desde já, pode-se dizer que esta prótese deve ser feita preferencialmente de material sintético, reticular e macro poroso para ficar em contato com a musculatura e de outro material laminar e micro poroso para ficar em contato com as vísceras. 


\section{REFERÊNCIAS}

1. Agarwal BB, Agarwal KA, Mahajan KC. Prospective double-blind randomized controlled study comparing heavy and lightweight polypropylene mesh in totally extraperitoneal repair of inguinal hernia: early results. Surg Endosc 2009, 23(2):242-247.

2. Bauer JJ, Salky BA, Gelernt IM, Kreel I. Repair of large abdominal wall defects with expanded polytetrafluoroethylene (PTFE). Ann Surg 1987, 206:765-769.

3. Bellón JM, Buján J, Contreras LA. Comparison of a new type of polytetrafluoroethylene patch (mycro mesh) and polypropylene prothesis (Marlex) for repair of abdominal wall defects. J Am Coll Surg 1996, 183:11-18.

4. Bellón JM, Contreras LA, Pascual G, Buján J. Evaluation of the acute scarring response to the implant of different types of biomaterial in the abdominal wall. J Mater Sci Mater Med 2000, 11(1):25-29.

5. Bellón JM, Jurado F, García-Moreno F, Corrales C, Carrera-San Martín A, Buján J. Healing process induced by three composite prostheses in the repair of abdominal wall defects. J Biomed Mater Res 2002, 63:182-190.

6. Bellón JM, Serrano N, Rodríguez M, Garcia-Honduvilla N, Pascual G, Buján J. Prótesis compuestas em las reparaciones de defectos de pared abdominal. Estúdio comparativo del empleo de barreras físicas y/o químicas. Cir Esp 2005, 77(6):351-356.

7. Burger JWA, Halm JA, Wijsmuller AR, Ten Raa S, Jeekel J. Evaluation of new prosthetic meshes for ventral hernia repair. Surg Endosc 2006, 20(8):1320-1325.

8. Conze J, Rosch R, Klinge U, Weiss C, Anurov M, Titkowa S, Oettinger A, Schumpelick V. Polypropylene in the intra-abdominal position: influence of pore size and surface area. Hernia 2004, 8(4):365-372.

9. Danino AM, Malka G, Revol M, Servant JM. A scanning electron microscopical study of the two sides of polypropylene mesh (Marlex) and PTFE (Gore Tex) mesh 2 years after complete abdominal wall reconstruction. A study of 15 cases. Br J Plast Surg 2005, 58(3):384-388.

10. DeCherney AH, DiZerega GS. Clinical problem of intraperitoneal postsurgical adhesion formation following general surgery and the use of adhesion prevention barriers. Surg Clin North Am 1997, 77(3):671-688.

11.Demir U, Mihmanli M, Coskun H, Dilege E, Kalyoncu A, Altinli E, Gunduz B, Yilmaz B. Comparison of prosthetic materials in incisional hernia repair. Surg Today 2005, 35:223-227.

12. Deysine M. Hernia repair with expanded polytetrafluoroethylene. Am J Surg 1992, 163(4):422-424.

13. Gonzalez R, Rodeheaver GT, Moody DL, Foresman PA, Ramshaw BJ. Resistance to adhesion formation: A comparative study of treated and untreated mesh products placed in the abdominal cavity. Hernia 2004, 8:213-19.

14. Kapan S, Kapan M, Goksoy E, Karabicak I, Oktar H. Comparison of PTFE, pericardium bovine and fascia lata for repair of incisional hernia in rat model, experimental study. Hernia 2003, 7:39-43.

15. Klosterhalfen $B$, Junge $K$, Klinge $U$. The lightweight and large porous mesh concept for hernia repair. Expert Rev Med Device 2005, 2(1):103-117.
16. Lamb JP, Vitale T, Kaminski DL. Comparative evaluation of synthetic meshes used for abdominal wall replacement. Surgery 1983, 176:271-76.

17. Law NW. A comparison of polypropylene mesh, expanded polytetrafluoroethylene patch and polyglycolic acid mesh for the repair of experimental abdominal wall defects. Acta Chir Scand 1990, 156:759-762.

18. Mathews BD, Pratt $B L$, Pollinger HS, Backus $C L$, Kercher KW, Sing RF, Heniford BT. Assessment of adhesion formation to intraabdominal polypropylene mesh and polytetrafluoroethylene mesh. J Surg Res 2003, 114(2):126-132.

19. Nyhus LM, Bombeck T. Hernias. In: Sabiston Jr DC. Textbook of Surgery - The Biological Basis of Modern Surgical Practice. IgakuShoin/Saunders, 13a edição, Philadelphia, PA, 1986, 1231-1232.

20. O'Dwyer PJ, Kingsnorth NA, Molloy RG, Small PK, Lammers B, Horeyseck G. Randomized clinical trial assessing impact of a lightweight or heavy-weight mesh on chronic pain after inguinal hernia repair. Br J Surg 2005, 92:166-170.

21. Ray NF, Larsen Jr. JW, Stillman RJ, Jacobs RJ. Economic impact of hospitalizations for lower abdominal adhesiolysis in the Unites States in 1988. Surg Gynecol Obst 1993, 176:271-276.

22. Restrepo JFP. Hérnias abdominais. In: Coelho JCU. Aparelho Digestivo - Clínica e Cirurgia. Medsi, 2a edição, Rio de Janeiro, RJ, 1996, 1569-1570, 146.

23. Sher W, Pollack D, Paulides CA, Matsumoto T. Repair of abdominal wall defects: gore-tex vs. marlex graft. Am Surg 1980, 46(11):618-623.

24. Simmermacher RKJ, Schakenraad JM, Bleichrodt RP. Reherniation after repair of the abdominal wall with expanded polytetrafluoroethylene. J Am Coll Surg 1994, 178(6):613-616.

25. van't Riet $M$, de Vos van Steenwijk PJ, Bonthuis F, Marquet $\mathrm{RL}$, Steyerberg EW, Jeekel J, Bonjer J. Prevention of adhesion to prosthetic mesh: comparison of different barriers using an incisional hernia model. Ann Surg 2003, 237(1):123-128.

26. Vavrík J, Foltýnová V, Vítková I, Adámek S, Poucková P. Changes in abdominal wall after mesh implantation in rats. Med Sci Monit 2000, 6(3):476-479.

27. Weyhe D, Schmitz I, Belyaev O, Grabs R, Müller KM, Uhl W, Zumtobel V. Experimental comparison of monofile light and heavy polypropylene meshes: less weight does not mean less biological response. World J Surg 2006, 30:1586-1591.

28. Weyhe D, Schmitz I, Belyaev O, Grabs R, Müller KM, Uhl W, Zumtobel V. Experimental comparison of monofile light and heavy polypropylene meshes: less weight does not mean less biological response. World J Surg 2007, 31:867-868.

29.Zieren J, Paul M, Osei-Agyemano T, Maecker F, Müller JM. Polyurethane-covered dacron mesh versus polytetrafluoroethylene dualmesh for intraperitoneal hernia repair in rats. Surg Today 2002, 32:884-886. 